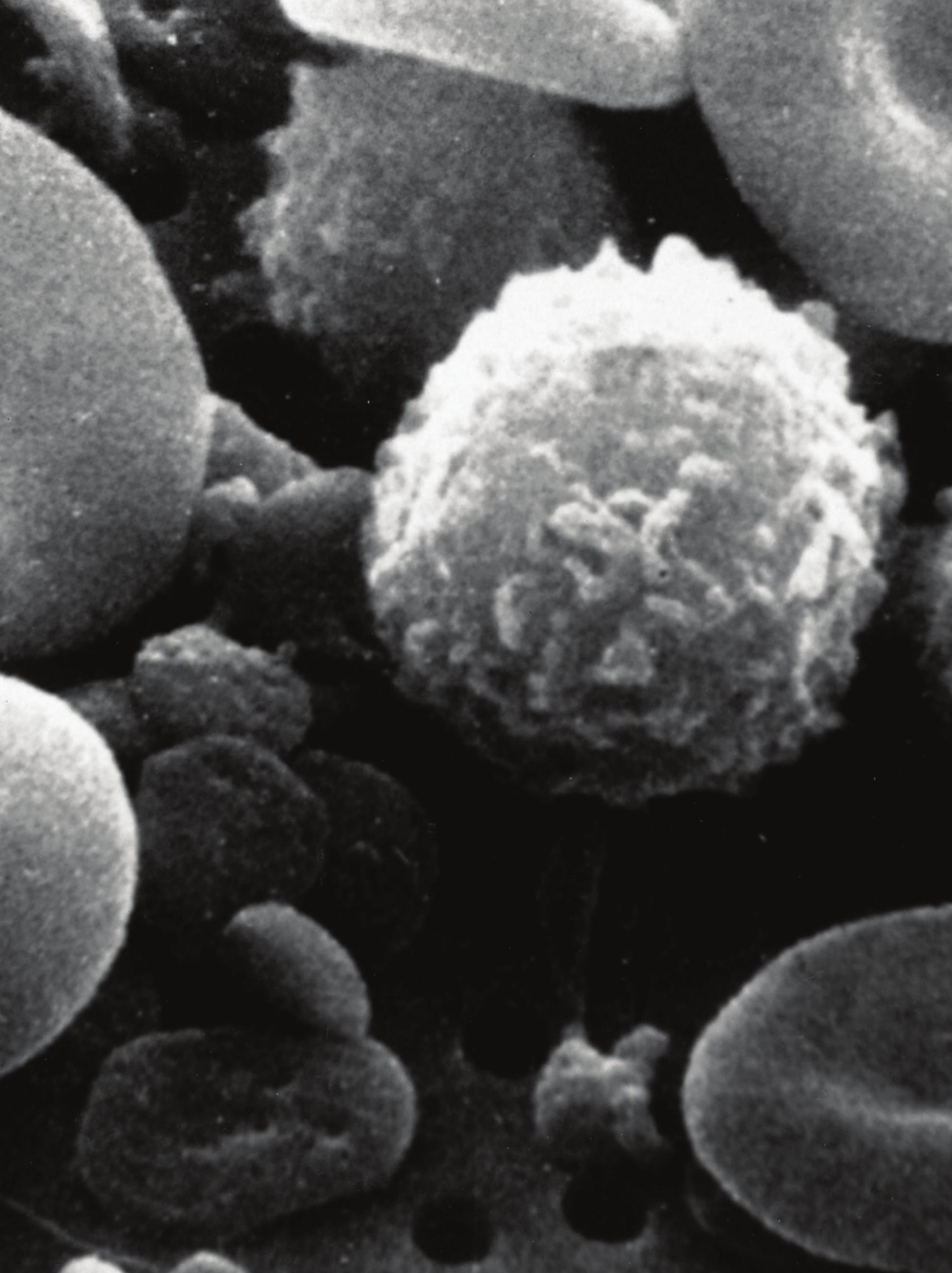

Image: Bruce Wetzel - Harry Schaefer, Wikimedia Commons 


$\begin{array}{lll}\text { Martin Iversen } & \begin{array}{l}\text { Section of Lung } \\ \text { Transplantation } \\ \text { Rigshospitalet Copenhagen } \\ \text { Denmark }\end{array} & \text { martin.iversen@dadlnet.dk }\end{array}$

\section{Immunosuppression for the non-transplant physician: what should you know?}

\section{Summary}

Immunosuppression is considered a difficult topic. In general, pulmonologists are experienced with anti-inflammatory treatment in the form of steroids and immunosuppressive treatment with moderate doses of methotrexate or azathioprine; some specialists treating patients with interstitial lung disease have experience with more immunosuppression and a larger number of drugs. Life-long intensive immunosuppression, as in lung transplant recipients, is a task for the specialist, but with more than 3,500 lung transplantations in the world annually (figure from the International Society for Heart and Lung Transplantation; www.ISHLT.org) many non-transplant pulmonologists will encounter lung transplant recipients, and will need some basic knowledge about immunosuppression. Also, an increasing number of patients with interstitial lung disease are treated with high-intensity immunosuppressive treatment.

This review is intended to be an introduction on general immunosuppression in patients with lung disease and lung transplant recipients. For a more comprehensive review the reader is advised to consult one or more of the large reviews published in recent years [1-4] or consult the Lung Transplantation issue of the European Respiratory Monograph (Issue 45; 2009) where a lot of background information on lung transplantation can be found.
Statement of Interest None declared. 


\section{General principles in immunosuppression}

The degree of immunosuppression cannot be measured directly and has to be inferred from the dose of drugs given, the concentration of drugs obtained in blood, or the general signs of over-immunosuppression such as repeated infections, and toxicity from drugs. In transplant recipients, the number and severity of acute rejections is the most important parameter to assess the level of immunosuppression in the first months after transplantation; however, this requires biopsies. Most lung transplant programmes have transbronchial biopsies at scheduled time points in the first 12 months. It is important to realise that acute rejections cannot be totally avoided. A balance must be found between an acceptable number of rejections and over-immunosuppression with infection. In spite of the complexity of the treatment there are some general rules that can be applied, shown in table 1. Most of what is known in immunosuppression is based on clinical experience and extrapolations from animal experiments. There are very few randomised clinical trials in lung patients.

Typical examples of low-grade immunosuppression would be patients with short-term high dose steroids because of exacerbation in asthma or chronic obstructive pulmonary disease or low-dose steroids and moderate-dose azathioprine or methotrexate in sarcoidosis patients. These patients would be slightly immunosuppressed but not so much as to influence all the decision making concerning the patient.

In lung transplant recipients or patients with interstitial lung disease receiving high intensity, long-term immunosuppression the situation is different and the treatment will influence your management of the patient in all aspects. A special problem arises with the use of polyclonal antibodies or monoclonal antibodies directed towards different cell populations and producing cell depletion of immunocompetent cells for very long periods, sometimes life-long. Some general principles for high-intensity immunosuppression can be stated as advice for the treating physician (table 2).

For lung transplant recipients there are further aspects to take into account because of the combination of immunosuppressive agents. Lung transplant recipients always receive one of the drugs cicloporine $[5,6]$ or tacrolimus [7, 8], belonging to the group of calcineurin inhibitors (CNI). CNIs are drugs with a relative specificity on T-lymphocytes, which are the key cells in acute cellular rejection in transplantation. By combining one $\mathrm{CNI}$ with an antimetabolite and prednisolone, a sufficient degree of immunosuppression can be obtained without too much general immunosuppression, so that the patient does not succumb to infection or drug toxicity. Both cyclosporine and tacrolimus are, however, strongly nephrotoxic and decrease measured

Table 1 General principles in immunosuppression

- With one drug in a moderate dose, or high dose short-term, you are safe

- With two drugs in low-moderate dose you are safe. With one of two in high dose there is risk of over-immunosuppression

- With three drugs there is always risk of over-immunosuppression irrespective of dose

- Usually a certain degree of immunosuppression can be obtained with different combinations of drugs

- Conventional drugs (steroids, antimetabolites and calcineurin inhibitors) have short-term effect (days to weeks)

- Biological drugs (polyclonal and monoclonal antibodies) have long-term effects (months to years) 
Table 2 Important points to consider in immunosuppressed patients

- Immunosuppression alters the patients response to acute disease, and reactions are blunted

- Patients have a suppressed reaction to infection and do not appear as ill as they are, and the usual clinical signs are not applicable

- Infection with opportunistic organisms such as fungi, pneumocystis and viruses is always a possibility

glomerular filtration rate shortly after transplantation [9] and most lung transplant recipients have substantially reduced renal function [10] which means that the patients do not tolerate dehydration and that, in many cases, drug doses must be reduced.

\section{Drugs used in immunosuppression}

The following is not a systematic review of all relevant facts but clinically important points for the various drugs are emphasised.

\section{Steroids}

Due to their broad anti-inflammatory and immunosuppressive qualities, steroids are used in nearly all lung transplant recipients; however, the maintenance dose is as low as $2.5-10 \mathrm{mg}$ daily. In treatment of acute rejections, doses of methylprednisolone 1,000 mg daily are given for 3 days followed by $40-50 \mathrm{mg}$ tapered to $5 \mathrm{mg}$ over 3 weeks. Whenever other drugs have to be lowered in dose or discontinued for a few days due to side effects, steroids can be temporarily increased to substitute the immunosuppressive action of other drugs.

\section{Antimetabolites}

The most commonly used antimetabolites are azathioprine and mycophenolatmophetil (MMF). Azathioprine is a well-known immunosuppressive drug. It is a prodrug which is metabolised to 6-mercaptopurin. The bioavailability is around $40 \%$ and plasma half-life is 2 hours; however, intracellular metabolites have much longer duration and are probably responsible for the main immunosuppressive action of the drug. Of interest is that the enzyme metabolizing 6-mercaptopurin has a genetic polymorphism [11, 12] with $89 \%$ of patients being fast metabolisers, around $11 \%$ having intermediate metabolism and a fraction of a percentage having no metabolism leading to severe accumulation and bone marrow depression. The dose is usually $1-$ $2 \mathrm{mg}$ per $\mathrm{kg}$ body weight but often needs to be lowered due to side effects. An important interaction is with allopurinol which inhibits metabolism of azathioprine to such a degree

Table 3 Important points in immunosuppression in lung transplantation

- Most patients require three drugs

- One calcineurin inhibitor must always be included in the treatment

- The dose of each drug can be changed so that the overall immunosuppressive effect is the same

- Most patients have poor renal function and do not tolerate dehydration 
that the dose must be lowered to $25 \%$ or the drug cannot be used.

MMF is a prodrug [13] with a bioavailability of $90 \%$ and a selectivity for lymphocytes, because other cells have a salvage pathway to escape the effects of the drug. It has strong immunosuppressive actions and the optimal dose in transplantation is 1,000 mg twice daily with CNI but 1,500 mg twice daily can be used without CNI. In some patients, the dose needs to be lowered, primarily due to leukopenia or gastrointestinal side-effects. MMF does not have the interactions or side-effects of azathioprine and can be used as an alternative drug. It is, however, much more expensive. Internationally, most lung transplant recipients receive MMF as antimetabolite, but there is no convincing evidence that there is any difference between the drugs.

\section{CNIs}

There are two drugs in this group, ciclosporin and tacrolimus, which are considered equal with regard to immunosuppression. Tacrolimus has a much higher potency per unit weight; $1 \mathrm{mg}$ of tacrolimus corresponds to $100 \mathrm{mg}$ of cyclosporine. They are both fungal metabolites with a moderate $(30 \%)$ bioavailability and varying absorption due to change in diet or gastrointestinal disturbance. Both are eliminated via cytochrome $450 \quad\left(\mathrm{CYP}_{3} \mathrm{~A}_{4}\right)$ and they have many important interactions that will necessitate change of dose. Before prescribing a new drug to a patient on $\mathrm{CNI}$, any interaction with $\mathrm{CNI}$ must always be checked.

The drugs are toxic and have a narrow therapeutic interval and are always used with therapeutic drug monitoring. After a change in dose, the new steady-state concentration will be reached after one week. The drugs are dosed twice daily and trough levels in the morning are used for guidance. Measurement of the specific phosphatase activity in Tlymphocytes inhibited by CNI is available but has not been demonstrated to be superior to measurement of drug concentrations.

Concentrations are kept high in the first months and gradually lowered thereafter. Typical concentrations of cyclosporine and tacrolimus are $200-250 \mathrm{ng} \cdot \mathrm{mL}^{-1}$ and 10$15 \mathrm{ng} \cdot \mathrm{mL}^{-1}$ in the first 3 months and maintenance concentration of $100-150 \mathrm{ng} \cdot \mathrm{mL}^{-1}$ and $5-10 \mathrm{ng} \cdot \mathrm{mL}^{-1}$ after 1 year. Individual patients have individual target levels; some patients have low target levels due to absence of rejections, which allows lowering of the concentrations, whereas other patients have low target levels due to side-effects.

CNIs have many serious side-effects and, as mentioned previously, the most important is short-term and long-term renal toxicity which is often dose limiting. Although the drugs have a selective cellular mechanism which mainly effects T-lymphocytes many more cell-types are affected and the drugs have many potentially serious side-effects affecting the central nervous system and peripheral nerves, muscular tissue, vessels, and other tissues. The immunosuppressive action and side-effects, especially renal toxicity, are similar for the two drugs, but tacrolimus seems to be more diabetogenic and it does not cause hirsutism as cyclosporine does.

If a patient has intolerable side-effects to one of the drugs, it is worthwhile to change to the other drug.

Because of the potency and toxicity, the drugs are rarely used outside lung transplant recipients in pulmonology and a transplant specialist should be consulted when changing the dose of these drugs.

\section{Proliferation inhibitors (PI)}

There are two drugs in this group, sirolimus [14] and everolimus [15] and like CNIs they are fungal metabolites though are even more toxic. They have a specific cellular action for immunosuppression $[16,17]$ which is different from CNIs and they have a strong synergistic effect when given with CNIs. The two drugs have similar actions but they have different pharmacokinetics, as sirolimus has a half-life of 62 hours and everolimus 28 hours. In addition to their immunosuppressive action, they have antiproliferative actions inhibiting proliferation of a number of cells leading to inhibition of the formation of granulation tissue. Their combined action also inhibits the growth of a number of tumours [18] and this is increasingly used in oncology.

In transplantation, their role is to be used in combination with $\mathrm{CNI}$ to allow $\mathrm{CNI}$ to be reduced to very low concentrations due to the synergistic effect. Because proliferation inhibitors (PI) in themselves are not nephrotoxic, this allows for improvement in renal function [19].

The drugs have similar and frequent sideeffects that are usually dose-related. Skin 
eruptions, peripheral oedema, bone marrow depression, and gastrointestinal disturbances are the most common. Of special interest is the occurrence of interstitial pneumonitis which occurs in $5 \%$ of patients at low dose but with increasing frequency at higher doses $[20,21]$.

\section{Polyclonal antibodies}

Polyclonal antibodies in the form of $\mathrm{T}$ lymphocyte antibodies [22] (Thymoglobuline) are mainly used for induction in lung transplantation and in some cases in treatment of repeated rejections. The drug produces severe depletion of T-lymphocytes and profound immunosuppression lasting for many months which is important to consider at a later stage.

\section{Monoclonal antibodies}

Interleukin-2 inhibitor (basiliximab) is used for induction treatment in lung transplantation. It does not produce cell depletion or long-lasting immunosuppression and has very few side-effects [23].

Anti-CD52 (alemtuzumab) was available until recently for induction treatment and treatment of recurrent rejections. It produces profound and long-lasting immunosuppression [24].

A number of monoclonal antibodies are now appearing and being used in rheumatology, oncology, gastroenterology, and neurology but few of those have yet been used in pulmonology. The tumour necrosis factor (TNF) inhibitor infliximab and other types of TNF inhibitors have been used in recalcitrant sarcoidosis. These drugs increase the risk of mycobacterial disease.

\section{Management of immunosuppression}

The usual lung transplant recipient is treated with three drugs, the most common combination is prednisolone $5 \mathrm{mg}$ daily, tacrolimus $1 \mathrm{mg}$ tvice daily, and MMF $1000 \mathrm{mg}$ twice daily. Concentrations of $\mathrm{CNI}$ are measured at regular intervals to ensure that the concentration is within the target level set for the patient. Every time the CNI dose is changed or a drug with a possible interaction with $\mathrm{CNI}$ is prescribed the $\mathrm{CNI}$ concentration is measured after two weeks. In addition to immunosuppressive drugs most transplant centres use life-long prophylaxis with sulfometoxazole/trimethoprim against pneumocystis infection.

The drugs in this combination can be changed because of side-effects so that the over-all level of immunosuppression is maintained. One $\mathrm{CNI}$ can be substituted for the other and azathioprine can be substituted for MMF. Prednisolone can be discontinued if there are important side-effects but most patients continue on low dose prednisolone.

In case of severe side-effects to CNI like renal failure or central nervous system effects a $\mathrm{CNI}$ can be combined with a $\mathrm{PI}$ and $\mathrm{CNI}$ concentrations can be lowered to a fraction of the usual target dose; e.g. $30 \mathrm{ng} / \mathrm{ml} \mathrm{com}$ pared to $130 \mathrm{ng} / \mathrm{ml}$ for ciclosporine. The $\mathrm{PI}$ must also be kept at a low concentration to avoid over-immunosuppression, e.g. 3-4 ng/ $\mathrm{ml}$ for sirolimus and everolimus in combination with a CNI. When using this strategy the antimetabolite must be lowered or discontinued.

\section{Some clinical emergency situations}

The following are general guidelines for some typical clinical situations but always call your transplant centre if the transplanted patient has a serious condition because the immunosuppression might influence the management of the patient. With the following guidelines you will be able to institute treatment in an emergency situation and contact your transplant centre subsequently.

\section{Renal failure}

This can occur due to overdosing or accumulation of CNI so always check the concentration. The most common cause, however, is dehydration due to illness or gastrointestinal disturbance as patients treated with CNI do not tolerate dehydration. Rehydrate the patient intravenously and lower $\mathrm{CNI}$ or discontinue CNI for 3-4 days if renal failure is severe.

\section{Abdominal condition}

This would include a number of conditions like infections or surgery for cancer or 
emergency surgery so that the patient will be unable to be on oral medications for some days. Steroids and CNI is essential but all other treatment can be discontinued for up to one week. A typical solution would be to give CNI intravenously in $25 \%$ of the oral dose and supplement with methylprednisolone $20 \mathrm{mg}$ daily. On this basic treatment the transplant patient can be maintained for some days until recovery of abdominal function.

\section{Severe infection}

Conditions like severe pneumonia, peritonitis, and sepsis necessitates acute change in immunosuppression. The usual step is to discontinue the antimetabolite, lower the CNI $25 \%$ and increase steroids from 5 to $10 \mathrm{mg}$. Often intravenous administration will be required. Observe that the usual clinical signs of infection often not are present in immunosuppressed patients.
Reference

1. Bhorade SM, Stern E. Immunosuppression for lung transplantation. Proc Am Thorac Soc 2009; 6: 47-53.

2. Hopkins PM, McNeil K. Evidence for immunosuppression in lung transplantation. Curr Opin Organ Transplant 2008; 13: 477-483.

3. Knoop C, Haverich A, Fischer S. Immunosuppressive therapy after human lung transplantation. Eur Respir J 2004; 23: 159-171.

4. Snell GI, Westall GP. Immunosuppression for lung transplantation: evidence to date. Drugs 2007; 67: 1531-1539.

5. Borel JF, Feurer C, Gubler HU, et al. Biological effects of cyclosporin A: a new antilymphocytic agent. Agents Actions 1976; 6: 468-475.

6. Lemaire M, Fahr A, Maurer G. Pharmacokinetics of cyclosporine: inter- and intra-individual variations and metabolic pathways. Transplant Proc 1990; 22: 1110-1112.

7. Kino T, Hatanaka H, Miyata S, et al. FK-506, a novel immunosuppressant isolated from a Streptomyces. II. Immunosuppressive effect of FK-506 in vitro. J Antibiot (Tokyo) 1987; 40: 1256-1265.

8. Staatz CE, Tett SE. Clinical pharmacokinetics and pharmacodynamics of tacrolimus in solid organ transplantation. Clin Pharmacokinet 2004; 43: 623-653.

9. Hornum M, Iversen M, Steffensen I, et al. Rapid decline in ${ }_{51} \mathrm{Cr}$-EDTA measured renal function during the first weeks following lung transplantation. Am J Transplant 2009; 9: 1420-1426.

10. Hornum M, Burton CM, Iversen M, et al. Decline in ${ }_{51} \mathrm{Cr}$-labelled EDTA measured glomerular filtration rate following lung transplantation. Nephrol Dial Transplant 2007; 22: 3616-3622.

11. Szumlanski C, Otterness D, Her C, et al. Thiopurine methyltransferase pharmacogenetics: human gene cloning and characterization of a common polymorphism. DNA Cell Biol 1996; 15: 17-30.

12. Weinshilboum RM, Sladek SL. Mercaptopurine pharmacogenetics: monogenic inheritance of erythrocyte thiopurine methyltransferase activity. Am J Hum Genet 1980; 32: 651-662.

13. Staatz CE, Tett SE. Clinical pharmacokinetics and pharmacodynamics of mycophenolate in solid organ transplant recipients. Clin Pharmacokinet 2007; 46: $13-58$.

14. Mahalati K, Kahan BD. Clinical pharmacokinetics of sirolimus. Clin Pharmacokinet 2001; 40: 573-585.

15. Kirchner GI, Meier-Wiedenbach I, et al. Clinical pharmacokinetics of everolimus. Clin Pharmacokinet 2004; 43: 83-95.

16. Gurk-Turner C, Manitpisitkul W, Cooper M. A comprehensive review of everolimus clinical reports: a new Mammalian target of rapamycin inhibitor. Transplantation 2012; 94: 659-668.

17. Nashan B. Review of the proliferation inhibitor everolimus. Expert Opin Investig Drugs 2002 Dec; 11: 1845-1857.

18. Dancey J. mTOR signaling and drug development in cancer. Nat Rev Clin Oncol 2010; 7: 209-219.

19. Gullestad L, Mortensen SA, Eiskjaer H, et al. Twoyear outcomes in thoracic transplant recipients after conversion to everolimus with reduced calcineurin inhibitor within a multicenter, open-label, randomized trial. Transplantation 2010; 90: 1581-1589.

20. Escudier B, Eisen T, Porta C, et al. Renal cell carcinoma: ESMO Clinical Practice Guidelines for diagnosis, treatment and follow-up. Ann Oncol 2012; 23: Suppl. 7, vii65-viiz1.

21. Exposito V, de Prada JA, Gomez-Roman JJ, et al. Everolimus-related pulmonary toxicity in heart transplant recipients. J Heart Lung Transplant 2008; 27: 797-800.

22. Merion RM, Howell T, Bromberg JS. Partial T-cell activation and anergy induction by polyclonal antithymocyte globulin. Transplantation 1998; 65: 1481-1489.

23. Onrust SV, Wiseman LR. Basiliximab. Drugs 1999; 57: 207-213.

24. Weaver TA, Kirk AD. Alemtuzumab. Transplantation 2007; 84: 1545-1547. 